\title{
The State of Art Survey on Micro cantilevers for MEMS Devices
}

\author{
Tanvi Chandel*, Balwinder Singh \\ ${ }^{I}$ Academic and Consultancy Services Division, Centre for development of advanced computing, \\ Mohali, India, 160071
}

\begin{abstract}
Arrival of Micro-electromechanical system (MEMS) in the last decade has been immense and ever growing. MEMS is an expanding and fast growing technology with an extensive range of applications. Micro cantilevers belongs to MEMS set of devices and are often used for detection of forces in Micromechanical Sensors(e.g. pressure sensors \& accelerometers) and probe for force sensor to atomic force microscope used as transducers, transport mechanisms, relays, switches, resonators etc. In this paper, the relative study and recent development of different micro cantilevers which differ in the material being used and in their construction are discussed and compared to each other based on various parameters.
\end{abstract}

Keywords: MEMS, Microcantilever, principles, shapes

\section{Introduction}

MEMS as the name suggests is a Micro-electromechanical System which comprises of mechanical elements, actuators, sensors \& (electronics \& electrical) devices miniaturized on a single silicon substrate. It is an emerging field with a vast range of applications. Cantilever is a wide ranging used component in micro system devices. It has been proven as an excellent platform for acute sensitive biological and chemical sensors. Micro cantilevers has enhanced and very popular due to its high selectively and sensitivity, flexibility and ease of fabrication on chip circuits. Also it has a wide dynamic range and is readily deployable into system. It is a beam having one end fixed and other end free. The load is carried by the beam to support where it is withstand by the moment and stress. The cantilever deflects when a force is applied exerted to the end. The length of the cantilever is greater as compare to its width with optimum thickness.

\subsection{Modes of operation of cantilever}

The cantilevers are operated either in Static Mode or in Dynamic Mode.

STATIC MODE: Due to load if generated, the displacement exists in the Cantilever, which is then measured and thus, this is the static mode.

DYNAMIC MODE: Due to change in stress of the cantilever concludes in a frequency change, which is then measured, thus when the cantilever oscillates at its natural frequency caused by external actuation, it is in dynamic state.

\subsection{Design parameters of Cantilever}

Predominantly, two equations are important to understand the behaviour of MEMS cantilevers.

The First Equation is STONEY's Formula which correlate cantilever end deflection " $\delta$ ” to exerted Stress ' $\sigma$ ' $[6]$.

$$
\delta=\frac{3 \sigma(1-V) E}{\left(\frac{\mathrm{L}}{\mathrm{t}}\right)^{2}}
$$

Where ' $V$ ' is Poisson's ratio, ' $L$ ' is beam length, ' $t$ ' is cantilever thickness, ' $E$ ' is Young's modulus. The second formula correlates the spring constant ' $\mathrm{K}$ ' to the cantilever dimensions \& material constant.

$$
\begin{array}{ll} 
& \mathrm{K}=\frac{\mathrm{F}}{\delta}=\frac{\mathrm{Ewh}^{3}}{4 \mathrm{~L}^{3}} \\
\text { Or } & \mathrm{K}=\frac{3 \mathrm{EI}}{\mathrm{L}^{3}} \\
\text { Where } & \mathrm{I}=\frac{\mathrm{wh}^{3}}{12}
\end{array}
$$

'F' is exerted Force, 'E' is Young's modulus, ' $\mathrm{I}$ ' is Moment of inertia, ' $\mathrm{L}$ ' is Beam length. The exerted force F is directly proportional to the bending of cantilever ' $\mathrm{z}$ ' \& spring constant ' $\mathrm{K}$ '.

$$
\text { Mathematically, } \quad \mathrm{F}=-\mathrm{kz}
$$




\section{Classification of cantilevers based on shapes}

\subsection{Rectangular shaped cantilever}

Many researches have been done on rectangular shaped cantilever [6], a MEMS based rectangular cantilever using COMSOL Metaphysics is made up of single crystal silicon having length 60E-6, breadth 10E-6, thickness $1.5 \mathrm{E}-6$ and simulated after being optimized with silicon dioxide on silicon substrate [6]. The Material being used is Silicon, Polysilicon \& Silicon nitride [5]:

Table 1: Chosen material properties used as film [5], [6]

\begin{tabular}{c|c|c|c|c}
\hline \multirow{2}{*}{ Material Properties } & \multicolumn{4}{|c}{ Material Names } \\
\cline { 2 - 5 } & $\mathbf{S i O}_{\mathbf{2}}$ & $\mathbf{S i}_{3} \mathbf{N}_{\mathbf{4}}$ & Poly-Si & $\mathbf{S i}$ \\
\hline Young's Modulus (Pa) & $70 \mathrm{E} 9$ & $250 \mathrm{E} 9$ & $160 \mathrm{E} 9$ & $0.2 \mathrm{E} 9$ \\
\hline Density(kg/m $\left.{ }^{3}\right)$ & 2200 & 3100 & 2320 & 2330 \\
\hline Poisson's Ratio & 0.17 & 0.23 & 0.22 & 0.33 \\
\hline
\end{tabular}

From the different material properties and evaluation done, it is found that, for simulation, $\mathrm{SiO}_{2}$ is the best analysis material because of availability of etching processes and ease of deposition. As per the simulation results[6],

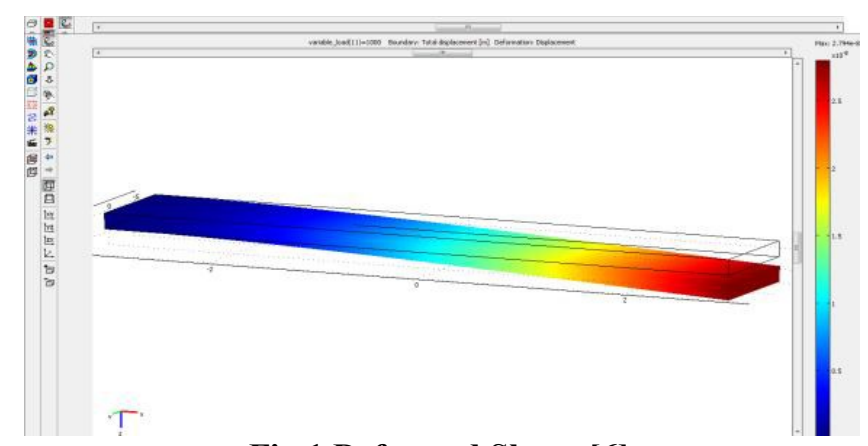

Fig.1 Deformed Shape [6]

The cantilever behaviour in expression of displacement with respect to variable load is shown in fig 3. This result demonstrates the Hook's law which states that the stress is directly proportional to Strain [6].

$$
\mathrm{F}=-\mathrm{kx}
$$

Where ' $\mathrm{F}$ ' is restoring force, ' $\mathrm{x}$ ' is displacement , ' $\mathrm{k}$ ' is spring constant.

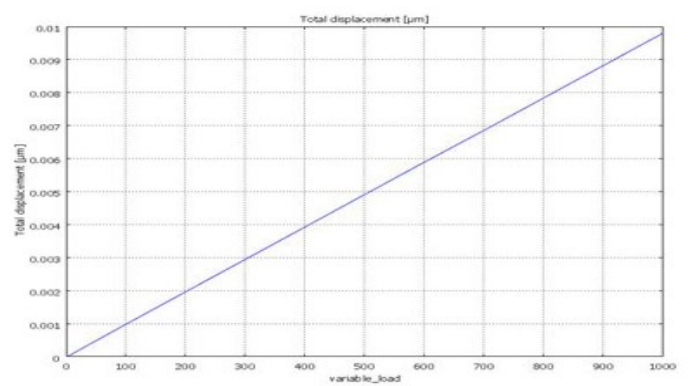

Fig.2 Cantilever Displacement Measurement [6]

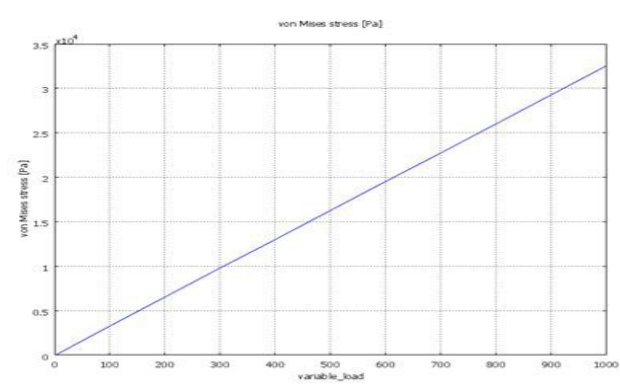

Fig.3 Cantilever Stress measurement [6]

\subsection{Triangular shaped cantilever} [11].

The triangular shaped cantilever which is simulated in ANSYS for strain distribution is shown below

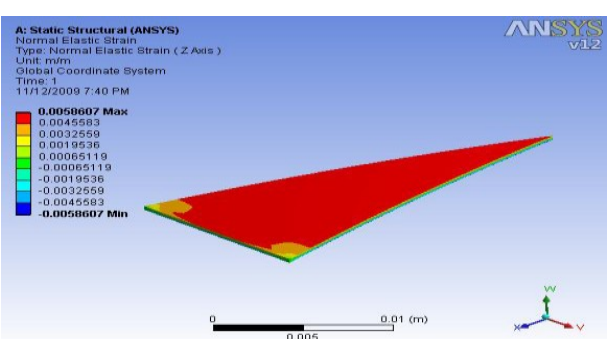

Fig.5 Stress Distribution for triangular Cantilever [11]

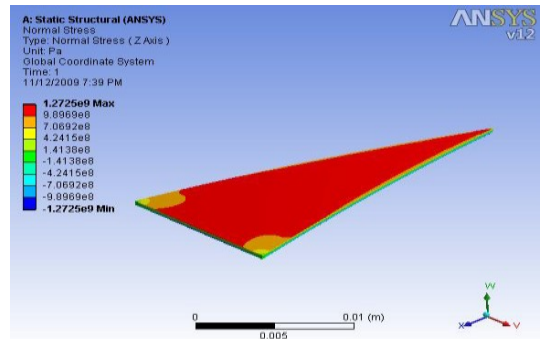

Fig.6 Strain distribution for Triangular cantilever [11] 
The materials used are polysilicon \& gold. The two layers of both the materials are sandwiched together. To avoid non specific interaction, polysilicon is used whereas gold layer allows the immobilization of antibodies by gold thiol interaction.

The resonance frequency in the dynamic mode of the cantilever is stated as [1]

$$
\mathrm{f}=\frac{1 \sqrt{\mathrm{k}}}{2 \pi \sqrt{\mathrm{m}_{\text {eff }}}}
$$

Where ' $\mathrm{k}$ ' is spring constant, ' $\mathrm{m}_{\text {eff }}$ ' is dynamic or effective mass. Therefore, the surface area of this shaped cantilever at any point has maximum value, thus has equal distribution for stress-strain.

\subsection{V-shaped cantilever}

In a V-Shaped Cantilever, no exact solution is well known. As an auxiliary approach, to calculate the natural fundamental frequencies, FEM Method is distinctly used [12]. The geometrical parameters used in FEM simulations are:-

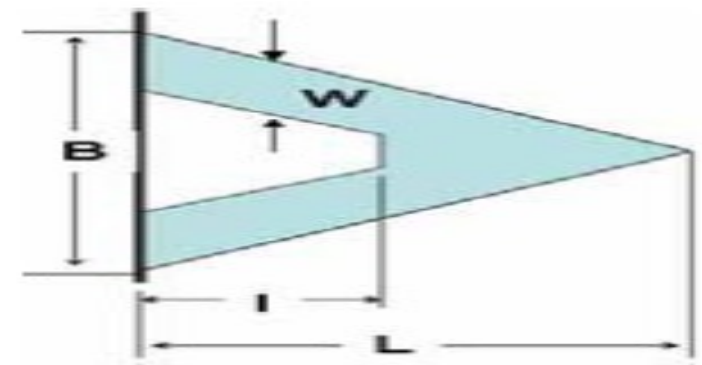

Fig .7 Cross sectional view of $\mathrm{V}$ - shaped AFM cantilever [12]

With the help of mesh module of COMSOL, it is attainable to extrude the meshes with thin thickness.

\subsubsection{Different types of ' $v$ ' shaped cantilevers}

The different types of V- Shaped Cantilevers are STS E Type, STS D Type, and STS F Type. Therefore, the first mode of STS F Type cantilever is

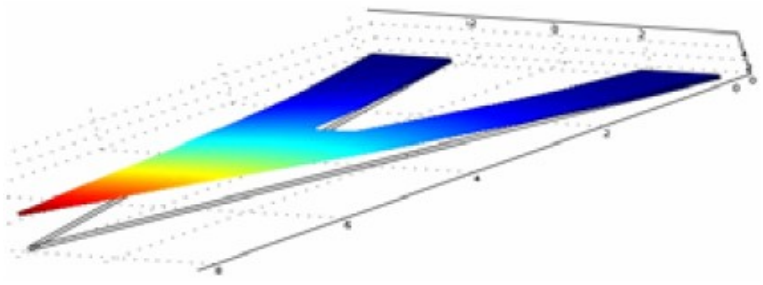

Fig. 8 Deformed Shaped corresponding to $1^{\text {st }}$ Vibration Mode [12]

Table 3: The Five FEM calculated frequencies are detailed as [12]

\begin{tabular}{|c|c|c|c|c|c|c|c|c|c|}
\hline & $\begin{array}{c}\mathbf{L} \\
(\mu \mathrm{m})\end{array}$ & $\begin{array}{c}1 \\
(\mu \mathrm{m})\end{array}$ & $\begin{array}{c}W \\
(\mu \mathrm{m})\end{array}$ & $\begin{array}{c}\text { B } \\
(\mu \mathrm{m})\end{array}$ & $\begin{array}{l}\text { Thickness } \\
\text { (nm) }\end{array}$ & $\begin{array}{c}\text { Mode } \\
\text { n }\end{array}$ & $\begin{array}{l}\text { Experimental } \\
\text { Frequency } \\
\text { (KHZ) }\end{array}$ & $\begin{array}{l}\text { Simulated } \\
\text { Frequency } \\
\text { (KHZ) }\end{array}$ & $\begin{array}{l}\text { Relative } \\
\text { Error } \\
(\%)\end{array}$ \\
\hline \multirow[t]{3}{*}{ STS C } & \multirow[t]{3}{*}{323} & \multirow[t]{3}{*}{235} & \multirow[t]{3}{*}{21} & \multirow[t]{3}{*}{227} & \multirow[t]{3}{*}{680} & 3 & 107.4 & 105.6 & $1.6 \%$ \\
\hline & & & & & & 4 & 204.6 & 203.6 & $0.6 \%$ \\
\hline & & & & & & 5 & 334.0 & 333.3 & $0.2 \%$ \\
\hline \multirow[t]{2}{*}{ STS D } & \multirow[t]{2}{*}{225} & \multirow[t]{2}{*}{133} & \multirow[t]{2}{*}{21} & \multirow[t]{2}{*}{158} & \multirow[t]{2}{*}{700} & 2 & 88.7 & 87.7 & $1.1 \%$ \\
\hline & & & & & & 3 & 233.2 & 234.4 & $0.5 \%$ \\
\hline \multirow[t]{2}{*}{ STS E } & \multirow[t]{2}{*}{128} & \multirow[t]{2}{*}{78} & \multirow[t]{2}{*}{18} & \multirow[t]{2}{*}{140} & \multirow[t]{2}{*}{560} & 2 & 193.4 & 194.7 & $0.8 \%$ \\
\hline & & & & & & 3 & 505.4 & 503.0 & $0.5 \%$ \\
\hline
\end{tabular}

Natural frequencies measurements and the simulated frequencies are compared and shown in fig 9. 


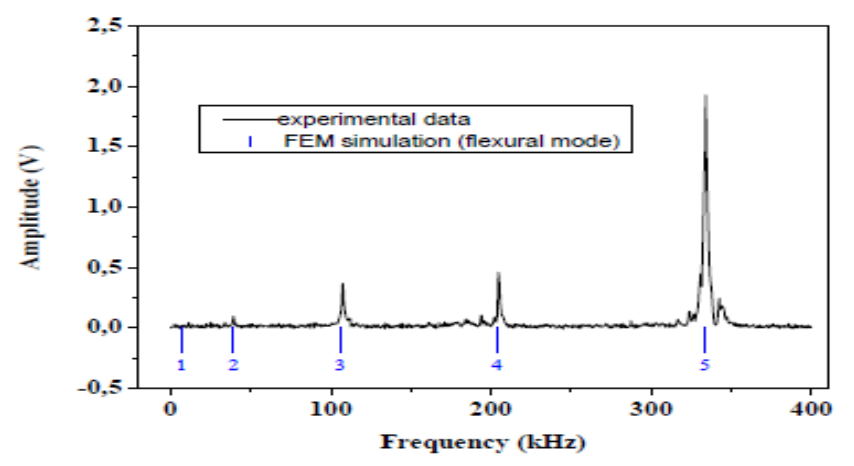

Fig. 9 Experimental Spectrum on a 'V-shaped Cantilever (STS C Type) [12]

The natural frequency measurements and simulated frequencies were compared, in resulting simulations only parameter that was adjusted is thickness of micro cantilever. The sensitivity of modified cantilever is given by a curved slope between relative variation of frequency and thickness of layer hence predicting the sensitivity of different geometrical dimensions [12].

\subsection{Trapezoidal shaped cantilever}

The trapezoidal shaped cantilever is shown in fig 10. The width of cantilever is $1250 \mu \mathrm{m}, \mathrm{L}$ denotes six different lengths from $1400 \mu \mathrm{m}$ to $1900 \mu \mathrm{m}$ with a step of $100 \mu \mathrm{m}$ [9].

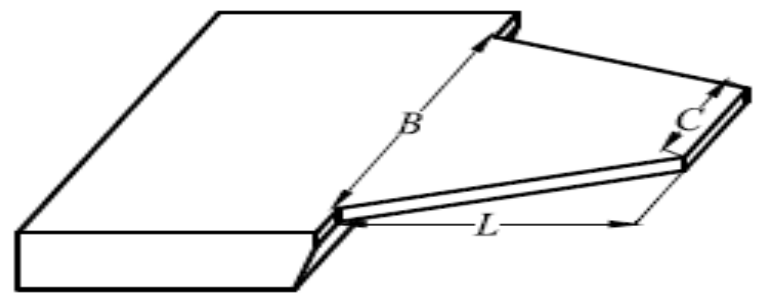

Fig.10 Trapezoidal cantilever [9] trapezoidal

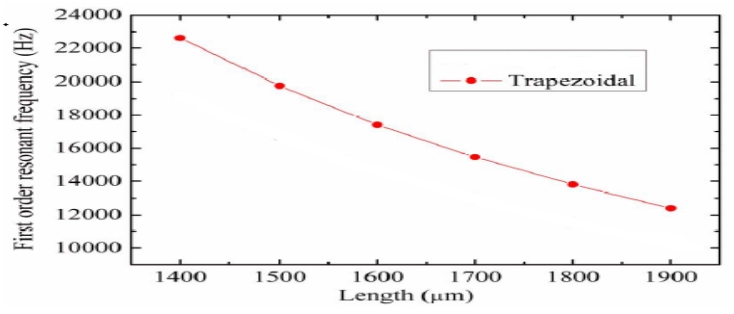

Fig.11The Curve of first order of frequencies of Cantilever to the length of cantilever [9]

In the Figure, B represents breadth of cantilever, L represents length of cantilever \& $\mathrm{C}$ represents the free end width of cantilever. The first order frequency of trapezoidal cantilevers with different lengths is studied through ANSYS Software in figure 11. Therefore, the trapezoidal cantilever has good performance due to its high resonant frequency \& Q factor. Thus, Results shows that viscosity sensor with trapezoidal cantilever has a fine accuracy to measure the viscosity of toluene[9].

\subsection{T-shaped micro cantilever}

The T-Shaped micro cantilever is proposed in which extra mass if included cause a change on the spring constant. This model enables to determine the optimal dimensions of geometry of structure in order to make the resonant frequency predominated by mass changes rather than the stiffness and therefore enhanced the sensitivity of the cantilever resonator. The three cantilevers are perpendicular to the substrate and are hold together with extra rectangular mass and are in flexural mode. At the backside, the multilayered piezoactuator with ceramic insulator is attached. The four piezoresistors measures the resonance frequency by connecting them in configuration of Wheatstone Bridge [8].

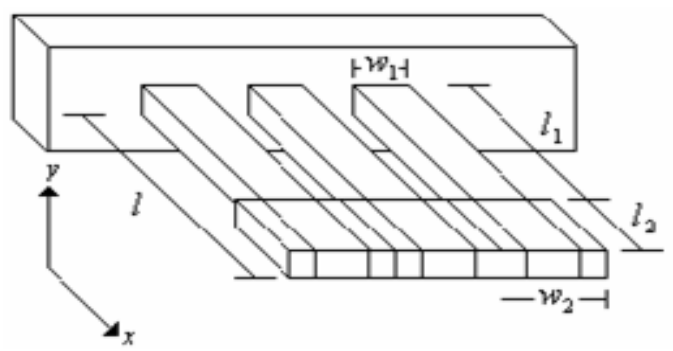

Fig.12 Diagram of T-Shaped Cantilever [8] 
Where $\mathrm{w}_{1}, \mathrm{l}_{1} \& \mathrm{~h}$ are width, length $\&$ thickness of each beam and $\mathrm{w}_{2}, \mathrm{l}_{2}$ are the width \& length of each extra mass. After the attachment of extra mass, the resonance frequency of each cantilever is [8]:

$$
\mathrm{f}=\frac{1}{2 \pi} \sqrt{\frac{\mathrm{k}+\Delta \mathrm{k}}{0.24 \mathrm{~m}_{1}+\mathrm{m}_{2}}}
$$

Where $\mathrm{k}+\Delta \mathrm{k}$ are spring constant when the mass is added, $\mathrm{m}_{2}$ is added mass and $0.24 \mathrm{~m}_{1}$ is the effective mass. After the attachment of mass $(\mathrm{k}+\Delta \mathrm{k})$, to calculate the spring constant, the equation of elastic curve is used as [8]:

$$
\frac{d^{2} y}{d x^{2}}=-\frac{F(x-L)}{E I}
$$

Where $\mathrm{E}$ is Young modulus of the material, I is Moment of inertia, F is applied force to the structure. Thus, Deflection is obtained as:

$$
\mathrm{y}_{\max }=\frac{4 \mathrm{~F}}{\mathrm{Eh}^{3}} \times\left(\frac{\mathrm{l}_{2}^{3}}{\mathrm{w}_{2}}+\frac{\mathrm{l}_{1}^{3}}{\mathrm{w}_{1}}+\frac{3 \mathrm{l}_{1}^{2} \mathrm{l}_{2}}{\mathrm{w}_{1}}+\frac{3 \mathrm{l}_{1} \mathrm{l}_{2}^{2}}{\mathrm{w}_{1}}\right)
$$

Therefore, to calculate $\mathrm{k}+\Delta \mathrm{k}$, the spring constant equation is used as:

$$
\mathrm{K}=\frac{\mathrm{F}}{\mathrm{y}_{\max }}
$$

Where ' $y_{\max }$ ' is the maximum displacement of spring, ' $\mathrm{F}$ ' is the force acting on the spring. Therefore, the new spring constant is shown as:

2.6 Pi-shaped micro cantilever

$$
\mathrm{k}+\Delta \mathrm{k}=\frac{\mathrm{Eh}^{3}}{4} \times \frac{\mathrm{w}_{1} \mathrm{w}_{2}}{\mathrm{l}_{2}^{3} \mathrm{w}_{1}+\mathrm{l}_{1}^{3} \mathrm{w}_{2}+3 \mathrm{l}_{1}^{2} \mathrm{l}_{2} \mathrm{w}_{2}+3 \mathrm{l}_{1} \mathrm{l}_{2}^{2} \mathrm{w}_{2}}
$$

The Pi-Shaped cantilever shape is given below. The simulation of pi-shaped cantilever is done on COMSOL as shown[1]:

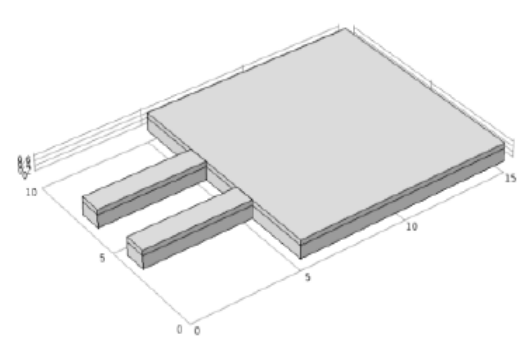

Fig.13 Pi Shaped Cantilever [1]

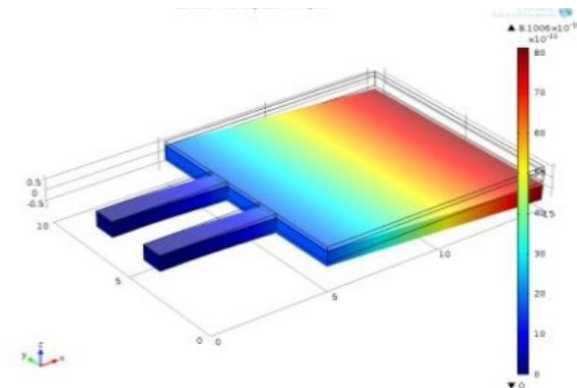

Fig.14 Stationary Study [1]

Thus, the output values of Eigen frequency and Displacement for Pi Shaped cantilever are observed as $1.8115^{*} 10-^{7}$ and $3.39693 * 10-^{21}[1]$.

\subsection{W-shaped micro cantilever}

The W- shaped cantilever is designed and the geometrical parameters are [14] :

Table 4 Geometrical Parameters of W shaped Cantilevers [14]

\begin{tabular}{c|c|c|c|c}
\hline Cantilever & $\mathbf{1 1}(\mathbf{m m})$ & $\mathbf{1 2 / 1 1}$ & $\boldsymbol{\alpha}(\mathbf{)}$ & $\mathbf{\beta}-\boldsymbol{\alpha}(\mathbf{)})$ \\
\hline W1 & 7 & 0.57 & 60 & 68.6 \\
\hline W2 & 6 & 0.54 & 45 & 56.8 \\
\hline W3 & 5 & 0.42 & 45 & 45.7 \\
\hline W4 & 4 & 0.52 & 30 & 46.2 \\
\hline W5 & 4 & 0.26 & 60 & 43.1 \\
\hline W6 & 3 & 0.51 & 60 & 68.6 \\
\hline W7 & 3 & 0.4 & 45 & 45.3 \\
\hline W8 & 3 & 0.28 & 60 & 48.2 \\
\hline W9 & 2 & 0.47 & 45 & 54.6 \\
\hline W10 & 2 & 0.28 & 60 & 51.7 \\
\hline
\end{tabular}

Certain Cantilever shapes have been designed made up of different lengths of rectangular beams [14]. 


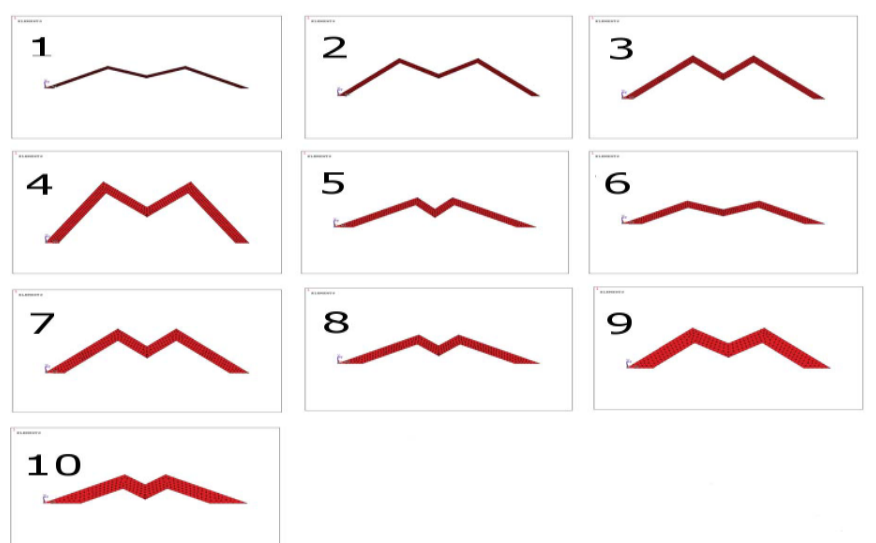

Fig. 15 Different geometries of designed W-shaped cantilevers [14]

There is a problem of titled tip during contact in scanning force microscopy. The measurement localization and stiffness of contact gets affected. These two parameters are critical for material characterization having large resolution. The $\mathrm{W}$ shaped cantilever stays the tip in the vertical direction and solves the above problems. Therefore, the $\mathrm{W}$ shaped Cantilevers can retain the tip vertical with suitable beam ratio during loading for forces whereas a bending of cantilever must occur.

\subsection{Piezoelectric cantilever}

\section{Classification of cantilevers based on principles}

The working principle of the piezoelectric is when stress is applied on a single layer or multiple layers of piezoelectric material such as PZT \& ZnO, electric field generates. Piezoelectric cantilevers are used in a range of applications such as in mass sensors, scanning tunnelling microscopy (STM), atomic force microscopy (AFM), accelerometers. In AFM applications, the resonating piezoelectric cantilevers are used and in others, it operates in a static mode.

\subsection{Electrostatic cantilever}

The working principle of Electrostatic cantilever is explained as, when the capacitor is formed between the cantilever surface and a fixed plate (i.e. counter electrode), the cantilever deflects and thus a change in capacitance can be measured. With this, the static \& dynamic deflection can be observed.

\subsubsection{Electrostatic micro cantilever model}

The Electrostatic Micro cantilever is modelled using conventor ware software build on a set of defined pseudo layers as shown[15]:

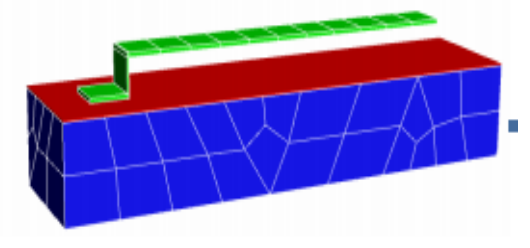

Fig.16 FEM mode of the micro cantilever [15]

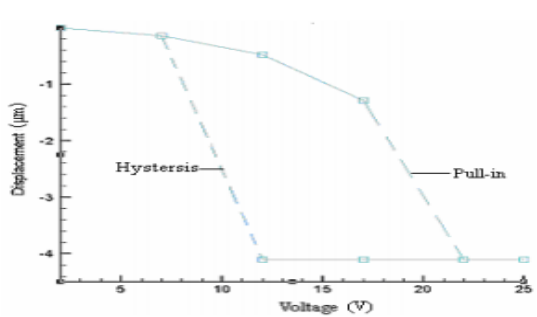

Fig.17 Hysteresis analysis for

Electrostatic Cantilever [15]

The displacement and stress analysis along with hysteresis and modal analysis has been done.

\subsection{Piezoresistive cantilever}

In a Piezoresisitive cantilever, the piezoresistive material used is single crystalline silicon. Sensitivity \& Noise are the two major parameters for Piezoresisitive cantilever. Two noises, low frequency $1 / \mathrm{f}$ noise and frequency independent Johnson noise are the supreme noise sources in this type of cantilever.

Firstly, the 1/f Noise in homogenous materials is dependent on the total number of carriers in resistors[2]. Therefore, Hooge in 1969 examined the evaluated results according to formula called Hoog's Formula, [3]

$$
\mathrm{S}_{\mathrm{VH}}=\frac{\alpha \mathrm{V}^{2}}{\mathrm{fN}}
$$

Where $\alpha$ is of range $10-^{7}$ to $10-^{3}$ which is a device independent - dimension parameter. 
The Johnson Noise arises from the random movement of electrons and can generate significant random movement at microscale dimensions. Thus, For a Resistance R, Johnson noise $\mathrm{S}_{\mathrm{VJ}}$, has spectral power, which is defined as [3]:-

$$
\mathrm{S}_{\eta}=4 \mathrm{kBTR}
$$

Where $T$ is temperature, $K_{B}$ is Boltzmann constant.

As the Force $\mathrm{F}$ acting on the tip of the cantilever, the cantilever bending will conclude in a resistance change. The senstivity of deflection is explained as a ratio of relative resistance change in cantilever to the deflection end of the cantilever $(\Delta z)$.

Therefore, the deflection senstivity for Rectangular cantilever is [3]:

$$
\frac{\Delta \mathrm{R}}{\mathrm{R}} / \Delta \mathrm{z}=\frac{3 \pi \mathrm{Et}\left(\mathrm{l}-\frac{\mathrm{L}}{2}\right)}{2^{2} \mathrm{1}^{3}}=\frac{3 \mathrm{Kt}\left(1-\frac{\mathrm{L}}{2}\right)}{2 \mathrm{1}^{3}}
$$

Where $\Delta \mathrm{z}$ is the vertical deflection in cantilever, $\mathrm{E}$ is the Young's modulus of material used in cantilever, $\mathrm{K}$ depicts the guage factor of the Piezoresistor, ' $t$ ' is the thickness of cantilever, ' $L$ ' is the length of cantilever, $\Delta \mathrm{R} / \mathrm{R}$ is the change in relative resistance.

Thus, Peizoresistive cantilever is used either in computation of properties of general cantilever such like mass and resonance frequency by a peizoresistive detection system or in the mesurement of stress/force such as deflection, accerelation and bending.

\subsection{Comparison among cantilevers \\ 1. Rectangular cantilever}

\section{Discussion}

The rectangular shaped cantilever is highly sensitive as it gives maximum displacement of $9.3887 * 10-^{19}$ for 50 antigens input mass [6]. It has a limitation of smaller strain effect in surface area, resulting in lesser strain-stress distribution.

\section{Triangular shaped cantilever}

As per simulation results, highest value of output power can be produced by triangular shaped cantilever. It has equal distribution for stress-strain, which has maximum value at any point on the surface of the shape.

\section{3. $V$ shaped cantilever}

For V-shaped cantilevers, in order to calculate fundamental natural frequencies, FEM method is used since there is no exact solution available.For the pupose of evaluting senstivity of cantilever as chemical transducers,an experimental study has been done.The natural frequency measurements and simulated frequencies were compared, in resulting simulations only parameter that was adjusted is thickness of microcantilever.The senstivity of modified cantilever is given by a curved slope between relative variation of frequency and thickness of layer hence predicting the senstivity of different geometrical dimensions.

\section{Trapezoidal shaped cantilever}

The trapizoidal shaped cantilever has good performance due to its high resonant frequency and Q factor. Thus, as per the simulation results, the application called viscosity sensor, with the use of trapizoidal cantilever, has a fine accuracy to measure the viscosity of toluene [9].

\section{T-shaped cantilever}

The T-shaped cantilever not only eradicates the limitation of small magnitude forces, but also increases the senstivity at the same time. As observed from simulations, T-shaped cantilever is very senstive and also has very low buckilng effect. However, with an increase in the thickness of the cantilever takes place, an equal decrease in the senstivity.

\section{Pi-shaped cantilever}

According to the paper [1], the value of displacement for Pi-shaped cantilever is $3.9693 * 10^{21}$ which is greater than triangular shaped cantilever displacement value $\left(1.0023 * 10{ }^{13}\right)$, while compared to the displacement value of pi-shaped cantilever, rectangular shaped cantilever displacement value $\left(9.3887 * 10^{9}\right)$ is lesser.

\section{W-shaped cantilever}

As per the simulations observed [14], the W-shaped cantilever has very low bending when a contact pressure is applied between tip and sample, which improves the localization of the measurement. 


\section{Conclusion}

Cantilever is a wide ranging used component in micro system devices. It has been proven as an excellent platform for acute sensitive biological and chemical sensors. The cantilevers are analysed on the basis of shapes and working principles. Thus, it has been observed that the Stress-Strain distribution in trapezoidal shaped cantilever is better, compared to the rectangular cantilever because the strain effect in surface area was larger than in rectangular cantilever. The triangular shaped cantilever has equal distribution for Stress-Strain, which has maximum value at any point. It has been observed that the rectangular shaped cantilever has maximum displacement as compared to other cantilevers. This review paper will be beneficial for MEMS researchers.

\section{References}

[1] Saranya R, Saranya K, Ceemati D, Chandra Devi K, Meenakshi Sundaram N, "Design of MEMS-based Microcantilever for Tuberculosis Detection," in Proc. Conf COMSOL, Banglore., 2013.

[2] Naeli, Kianoush, "Optimization of piezoresistive cantilevers for static and dynamic sensing applications," Ph.D. dissertation, Institute of Technology Georgia, School of Electrical and Computer Engineering, Georgia, 2009.

[3] Yu Xiaomei, Zhang Dacheng, Wang Wei, Li Ting, “A sensor platform based on piezoresistive cantilever," in Proc. IEEE Int. Conf Electron Devices and Solid-State Circuits., pp. 121-124, 2003.

[4] Mathad, Aditya G., and Rajendra M. Patrikar, "The Origin of Surface Stress experienced by a Micro-cantilever beam," in Proc. IEEE Int. Conf Students' Technology Symposium (TechSym)., pp 12-16, April 2010.

[5] Reddy, V. Mounika, and GV Sunil Kumar, "Design And Analysis of Microcantilevers With Various Shapes Using COMSOL Multiphysics Software," IJETAE Trans. vol. 3, issue 3, pp. 294-299, March 2013.

[6] Arora, Suryansh, Arti Arora Sumati, and P. J. George, "Design of MEMS based microcantilever using comsol multihysics," IJAER Trans., vol. 7, no.11, 2012.

[7] Jain, Vinod, and Saurav Verma, "Design and characteristics comparison of MicroCantilever for integrated sensing applications," in Proc. IEEE Int. Conf Advances in Technology and Engineering (ICATE)., pp. 1-4, 2013.

[8] Margarita Narducci, Eduard Figueras, Isabel Gràcia, Luis Fonseca, Joaquin Santander, Carles Cané, "Modeling of T-Shaped Microcantilever Resonators," in Proc. Int. Joint Conf., 2008.

[9] Longqi Xu, Guiming Zhang, Libo Zhao, Yulong Zhao, Zhuangde Jiang, Rahman-hebibul, "A fluid viscosity sensor with resonant trapezoidal micro cantilever," in Proc. IEEE Int. Symposium Assembly and Manufacturing (ISAM)., pp. 311-314, 2013.

[10] Gui-Ming Zhang, Li-Bo Zhao, En-Ze Huang, Guo-Ying Yuan, Yong Li, Zhi-Gang Liu and Zhuang-De Jiang, "On dynamic behavior of triangular shaped cantilevers in MEMS sensors: Effect of curvature," in Proc. Tenth IEEE Int. Conf Solid-State and Integrated Circuit Technology (ICSICT)., 2010.

[11] Shebeeb, Akeel, and Hanim Salleh, "Effect of cantilever shape on the power output of a piezoelectric bimorph generator," in Proc. IEEE Int. Conf Semiconductor Electronics (ICSE)., pp. 275-278, 2010.

[12] Louarn, G., M. Collet, and S. Cuenot, "Modeling of Different Shaped Micro-cantilevers Used as Chemical Sensors," in Proc. Conf COMSOL, Paris., 2006.

[13] Sader, John Elie. "Parallel beam approximation for V-shaped atomic force microscope cantilevers." Review of Scientific Instruments 66.9 (1995): 4583-4587.

[14] Julian Le Rouzic, Pascal Vairac, Bruno Cavallier, and Bernard Cretin, "W-Shaped Cantilevers for Scanning Force Microscopy," IEEE Trans. Sensors., vol. 13, no. 4, pp. 1340-1346, 2013.

[15] Liu, Fengli, and Yongping Hao, "Characteristic analysis and simulation for electrostatic micro-cantilever," in Proc. IEEE Int. Conf Information and Automation (ICIA)., pp. 1498-1502, 2008. 